\title{
Forecasting the Short-Term Traffic Flow in the Intelligent Transportation System Based on an Inertia Nonhomogenous Discrete Gray Model
}

\author{
Huiming Duan, ${ }^{1,2}$ Xinping Xiao, ${ }^{1}$ and Lingling Pei $^{3}$ \\ ${ }^{1}$ College of Science, Wuhan University of Technology, Wuhan 430070, China \\ ${ }^{2}$ College of Science, Chongqing University of Posts and Telecommunications, Chongqing 400065, China \\ ${ }^{3}$ School of Business Administration, Zhejiang University of Finance \& Economics, Hangzhou 310018, China \\ Correspondence should be addressed to Xinping Xiao; xiaoxp@whut.edu.cn
}

Received 15 April 2017; Accepted 28 May 2017; Published 3 July 2017

Academic Editor: Bo Zeng

Copyright (C) 2017 Huiming Duan et al. This is an open access article distributed under the Creative Commons Attribution License, which permits unrestricted use, distribution, and reproduction in any medium, provided the original work is properly cited.

\begin{abstract}
The traffic-flow system has basic dynamic characteristics. This feature provides a theoretical basis for constructing a reasonable and effective model for the traffic-flow system. The research on short-term traffic-flow forecasting is of wide interest. Its results can be applied directly to advanced traffic information systems and traffic management, providing real-time and effective traffic information. According to the dynamic characteristics of traffic-flow data, this paper extends the mechanical properties, such as distance, acceleration, force combination, and decomposition, to the traffic-flow data vector. According to the mechanical properties of the data, this paper proposes four new models of structural parameters and component parameters, inertia nonhomogenous discrete gray models (referred to as INDGM), and analyzes the important properties of the model. This model examines the construction of the inertia nonhomogenous discrete gray model from the mechanical properties of the data, explaining the classic NDGM modeling mechanism in the meantime. Finally, this paper analyzes the traffic-flow data of Whitemud Drive in Canada and studies the relationship between the inertia model and the traffic-flow state according to the data analysis of the traffic-flow state. A simulation accuracy and prediction accuracy of up to 0.0248 and 0.0273 , respectively, are obtained.
\end{abstract}

\section{Introduction}

Traffic-flow theory is the basic theory of the intelligent transportation system, that is, the use of mathematical and mechanical laws to study the laws of road traffic-flow theory [1]. By analyzing the relationship between the parameters of the traffic-flow system, it can seek to establish the most rational model to analyze the changes in traffic flow [2], providing a theoretical basis for rational planning and efficient traffic management. The study of traffic-flow theory promotes the interdependence and interaction of dynamics, applied mathematics, fluid mechanics, and traffic engineering.

Traffic-flow short-term forecasting for the intelligent transportation system to provide traffic information is an important basis for traffic analysis [3, 4] and control [5]. Short-term traffic-flow forecasting has been widely researched by scholars at home and abroad, who have obtained many research results [6-8], and many theories and methods $[9,10]$ have been applied to the study of shortterm traffic forecasting. The results of this study can be applied directly to the advanced traffic information system and traffic management system, which can provide realtime and effective information for walkers, realize the route planning, reduce the travel time of the traveler, alleviate road congestion, reduce pollution, save energy, and so on. Trafficflow forecasting is also based on the dynamic acquisition of traffic-flow time-series data to predict the future traffic-flow status data.

The traffic-flow characteristics can be described by the traffic-flow state, and the traffic flow exhibits different characteristics in different states. In the study of urban traffic-flow parameter models, the traffic state is divided into free flow, congested flow, and jam flow. Usually, the traffic-flow rate, speed, and occupancy rate are considered as parameters of the 
resulting traffic state. The interval and forecast period of timeseries data for short-term traffic-flow parameters are shorter, usually within 15 minutes. There are many methods for shortterm traffic-flow forecasting: chaos theory [11], time series $[12,13]$, neural networks $[14,15]$, nonparametric regression $[16,17]$, gray prediction [18], and other methods $[19,20]$.

However, the short-term traffic-flow system has a large degree of similarity with the fluid system with respect to basic dynamic characteristics and, at the same time, a high degree of uncertainty. It is difficult to accurately grasp the roles of the system factors and mechanisms due to poor information. If the time interval for collecting traffic is 5 minutes, only 12 groups of data are obtained in one hour, resulting in a small sample size. Therefore, it is reasonable to study the inertial gray model by using the gray system with less data and a poor information system combined with the mechanical properties of traffic-flow data.

The gray system theory was put forward by Deng [21]. After 30 years of development, a framework of system analysis and evaluation [22], model prediction [23-25], and decision control [26] has been established as the main technical system. The gray prediction model is the core component of gray system theory. Since its introduction, the gray prediction model has been widely studied and continuously developed and optimized [27-30]. $\mathrm{GM}(1,1)$, as the core gray prediction model, has also been improved $[25,31,32]$ and has been widely used in various fields. However, in practical applications and the theoretical research process, $\operatorname{GM}(1,1)$ is not fully suitable for fitting homogenous exponential series. The problem of transforming the $\operatorname{GM}(1,1)$ from discrete form to continuous form is solved by the proposed discrete gray model [33]. At the same time, many scholars have extended the properties and optimization of model parameters. However, the discrete gray model, like the classical $\operatorname{GM}(1,1)$, can only solve the problem of exponential growth order, and sequences with exponential growth are very rare in real life; comparatively speaking, more original sequence data conform to nonexponential growth laws. The discrete gray model of the approximate nonhomogenous exponential sequence extends the application range of the discrete model to approximate nonhomogenous exponential sequences [34], which enhances the applicability of the discrete gray model.

However, the gray prediction model of the classical $\mathrm{GM}(1,1)$, discrete gray model (DGM), and approximate nonhomogenous discrete gray model (NDGM) is used as the modeling mechanism of the least-squares method. These models do not describe the modeling process from the point of view of the data. Professor Deng proposed the inertia $\operatorname{GM}(1,1)$ in $[21]$, emphasizing that inertia is the quality of the material mass of a temperament, which is the abstract amount that has to be considered when researching material movement and thought movement. At the same time, he suggested that the data are generated by the thought movement in [35], and the value of the thinking process is much greater than the value of a certain function. It can be said that the number of sequences $X^{(0)}$ is the formation of thinking or things and that the sequence in different minds and the processes of forming different things have different meanings. He noted that, in the
$\operatorname{GM}(1,1), x^{(0)}(k)$ can reflect the velocity in mechanics, the accumulating generation operator (AGO) $x^{(1)}(k)$ can reflect the deposition of this process, and $z^{(1)}(k)$ can reflect the background, while at the same time representing the inertia $\operatorname{GM}(1,1)$ from the force resolution of the data. Traffic-flow theory and fluid systems have a high degree of similarity, as they both have the same basic dynamic characteristics.

Therefore, this paper introduces the basic concepts and properties of mechanics distance, acceleration, force combination, and resolution in traffic-flow data and studies the inertia model, which is adapted to short-term traffic forecasting. At the same time, the model structure clearly shows the formation process of the INDGM using understandable structure parameters and manifestation of component parameters. This model is also closely related to the classical NDGM, and the modeling mechanism of the classical NDGM from the mechanical decomposition of the data is illustrated. Finally, the paper analyzes the state data of traffic flow and appropriately selects the inertia model for traffic-flow data of Whitemud Drive in Canada, which can effectively improve the simulation and forecasting effect of short-term traffic flow.

This paper is organized as follows. In Sections 2, the basic concepts and properties of mechanics in the data vector are introduced. In Sections 3, the NDGM is introduced; the INDGM is put forward using the mechanics decomposition of the data, and an important property of the model is studied. In Sections 4, traffic-flow data from Canada is used for the fitting analysis in the empirical study. The conclusion of this study is discussed in Section 5.

\section{Basic Concepts and Properties of Mechanics in the Data Column}

Inertia is the temperament of the mass quality of the reaction material, and it is also the property of the energy system. The social, economic, technical, military, transportation, ecology, and agriculture are generalized energy systems. This section mainly introduces the basic concepts and mechanical properties of the mechanics in the data column.

Definition 1 (see [21]). Regarding distance, the following definitions are given:

(1) The measure of the position difference between two points is called distance.

(2) Let $\mathbb{F}$ be a proposition. The distance measure under $\mathbb{F}$ is the journey length.

Definition 2 (see [21]). Assume that the sequence $X^{(0)}=$ $\left(x^{(0)}(1), x^{(0)}(2), \ldots, x^{(0)}(n)\right)$ is an original data sequence under equal-interval proposition $\mathbb{F}$. If $\alpha(k)=x^{(0)}(k)-x^{(0)}(k-$ $1)$, the sequence $\alpha=(\alpha(2), \alpha(3), \ldots, \alpha(n))$ is an acceleration sequence under equal-interval proposition $F$. Let sgn $y(k)$ be the symbol of data $y(k)$; that is,

$$
\begin{aligned}
& \operatorname{sgn} y(k)=+1, \quad y(k) \geq 0, \\
& \operatorname{sgn} y(k)=-1, \quad y(k)<0 .
\end{aligned}
$$


Then, let $\beta$ be a nonnegative real number. $f(k)$ is the number consistent with $\alpha(k)$ if the following are satisfied:

(1) $\alpha(k)=\beta f(k), \forall k \in K, K=\{1,2, \ldots, n\}$,

(2) $x^{(0)}(k)=\beta f(k)-x^{(0)}(k-1)$,

(3) $\operatorname{sgn} f(k)=\operatorname{sgn} \alpha(k)$,

(4) $f(k)=$ Value $(k)$, for $\mathfrak{R}$,

where $f(k)=$ value $(k)$ under criterion $\mathfrak{R}$.

Let $\mathbb{F}$ be the proposition. The distance measure under $\mathbb{F}$ becomes as follows:

(1) $\beta$ is called the incentive coefficient of the sequence $X^{(0)}$

(2) $m$, which is the inverses of $\beta$, is called the inertial coefficient of the sequence $X^{(0)}$, where $m=1 / \beta$,

(3) $f(k)$ is called the external force of the sequence $X^{(0)}$ at the $k$ th moment (zone).

Definition 3 (see [21]). Let $X^{(0)}$ be the original sequence and let $\alpha$ be the acceleration sequence of $X^{(0)}$.

(1) If the external force $f(k)$ of $X^{(0)}$ at point $k$ satisfies $f(k)=\alpha(k)$, then $\beta=1$. This relationship is called the unit-incentive relationship, and the external force sequence $f$ under $\beta=1$ is called the external force sequence of unit incentive.

(2) If $\alpha(k)=q f(k)$ under the criterion $\mathfrak{R}$, that is, $\beta=q$, then we call the relationship

$$
\begin{aligned}
& \beta=\frac{\alpha(k)}{f(k)} \Longrightarrow \\
& \beta=\frac{q f(k)}{f(k)} \Longrightarrow \\
& \beta=q
\end{aligned}
$$

the incentive relationship corresponding to the incentive external force sequence $q$.

Definition 4 (see [21]). Let $X^{(0)}$ be the original sequence and let $\alpha$ be the acceleration sequence of $X^{(0)}$. Then

$$
\begin{aligned}
X^{(0)} & =\left(x^{(0)}(1), x^{(0)}(2), \ldots, x^{(0)}(n)\right), \\
\alpha & =(\alpha(2), \alpha(3), \ldots, \alpha(n)), \\
\alpha(k) & =x^{(0)}(k)-x^{(0)}(k-1),
\end{aligned}
$$

$\forall k \in K, K=\{1,2, \ldots, n\}$
Let $\beta \in\{1, q\}$ be the incentive and let $M_{f}$ be the transformation $M_{f}: \alpha \stackrel{\beta}{\rightarrow} f$, where

$$
\begin{aligned}
\alpha & =(\alpha(2), \alpha(3), \ldots, \alpha(n)), \\
f & =(f(2), f(3), \ldots, f(n)), \\
M_{f}(\alpha(k)) & =f(k), \\
f(k) & =\beta^{-1} \alpha(k),
\end{aligned}
$$

$\forall k \in K$,

and then $M_{f}$ is called the force transformation of data $x(k)$ and

$$
\begin{aligned}
f & =(f(2), f(3), \ldots, f(n)) \\
& =\left(\beta^{-1} \alpha(2), \beta^{-1} \alpha(3), \ldots, \beta^{-1} \alpha(n)\right) .
\end{aligned}
$$

Definition 5 (see [21]). Let $X^{(0)}$ be the original sequence and let $\alpha$ be the acceleration sequence of $X^{(0)}$. Suppose that the external force sequence $f$ under incentive $\beta$ satisfies

$$
M_{f}: \alpha \stackrel{\beta}{\longrightarrow} f, \quad f=(f(2), f(3), \ldots, f(n)) ;
$$

then

(1) $\Phi(k)$ is called the first-order combination of $f$, where $\Phi(k)=\sum_{i=2}^{k} f(i)$,

(2) $F(k)$ is called the second-order combination of $f$, where

$$
\begin{aligned}
& F(k)=\sum_{i=2}^{k} \Phi(i), \\
& F(1)=0,
\end{aligned}
$$

(3) $F_{\Sigma}(k)=\sum_{i=2}^{k} F(i)$ is called the third-order combination of $f$, where

$$
\begin{aligned}
& F(k)=\sum_{i=2}^{k} \Phi(i), \\
& F(1)=0,
\end{aligned}
$$

(4) $\left\{X^{(0)}, \beta,\{F\}\right\}$ is called the force space ( $\{F\}$ for short), where

$$
\{F\}=\left\{f(i), \Phi(i), F(i), F_{\Sigma}(i)\right\},
$$

$f(i)$ is called the first-order force element, $\Phi(i)$ is called the second-order force element, and $F(i)$ is called the third-order force element.

Definition 6 (see [21]). $x^{(0)}(k)$ of the original sequence $X^{(0)}, \operatorname{AGO} x^{(1)}(k)$ (Accumulating Generation Operator), and average sequence $Z^{(1)}(k)$, addressed as all order element, constitute the decomposition transform of data. 
Definition 7 (see [21]). Let $X^{(0)}$ be the original sequence, let $\operatorname{AGO} x^{(1)}(k)$ of $X^{(0)}$ be $X^{(1)}$, and let the average sequence of $X^{(1)}$ be $Z^{(1)}$,

$$
\begin{aligned}
X^{(0)} & =\left(x^{(0)}(1), x^{(0)}(2), \ldots, x^{(0)}(n)\right), \\
X^{(1)} & =\left(x^{(1)}(1), x^{(1)}(2), \ldots, x^{(1)}(n)\right), \\
x^{(1)}(k) & =\sum_{m=1}^{k} x^{(0)}(m), \\
Z^{(1)} & =\left(z^{(1)}(1), z^{(1)}(2), \ldots, z^{(1)}(n)\right), \\
z^{(1)}(k) & =0.5 x^{(1)}(k)+0.5 x^{(1)}(k+1) .
\end{aligned}
$$

Then one has the following:

(1) If transformation $M_{x^{(0)}}$ satisfies $M_{x^{(0)}}\left\{x^{(0)}(k)\right.$, $\left.x^{(0)}(1)\right\}=\Phi(k)$, then the inverse transformation $M_{x^{(0)}}^{-1}$ is called the decomposition transform of $x^{(0)}(k)$.

(2) If transformation $M_{x^{(1)}}$ satisfies $M_{x^{(1)}}\left\{x^{(1)}(k)\right.$, $\left.x^{(0)}(1)\right\}=F(k)$, then the inverse transformation $M_{x^{(1)}}^{-1}$ is called the decomposition transform of $x^{(1)}(k)$.

(3) If transformation $M_{z^{(1)}}$ satisfies $M_{z^{(1)}}\left\{z^{(1)}(k)\right.$, $\left.x^{(0)}(1)\right\}=\psi(k), \psi(k)=F(k-1)+0.5 \Phi(k)$, then the inverse transformation $M_{z^{(1)}}^{-1}$ is called the decomposition transform of $z^{(1)}(k)$.

(4) $M=\left\{M_{f}, M_{x^{(0)}}, M_{x^{(1)}}, M_{z^{(1)}}\right\}$ is called the forcetransformation set.

If $M_{f}$ satisfies $M_{x^{(0)}}\left\{x^{(0)}(k), x^{(0)}(1)\right\}=\Phi(k)$, then the inverse transformation $M_{x^{(0)}}^{-1}$ is called the decomposition transform of $x^{(0)}(k)$.

According to [21], theorems related to the forcedecomposition transform can be stated as follows.

Theorem 8 (see [21]). (1) Let $M_{x^{(0)}}^{-1}$ be the decomposition transform of $x^{(0)}(k)$,

$$
M_{x^{(0)}}\left\{x^{(0)}(k), x^{(0)}(1)\right\}=\Phi(k) .
$$

Then

$$
\begin{aligned}
\beta^{-1}\left(x^{(0)}(k)-x^{(0)}(1)\right)= & \Phi(k), \\
& x^{(0)}(k)=\beta \Phi(k)+x^{(0)}(1) .
\end{aligned}
$$

(2) Let $M_{x^{(1)}}^{-1}$ be the decomposition transform of

$$
x^{(1)}(k), M_{x^{(1)}}\left\{x^{(1)}(k), x^{(0)}(1)\right\}=F(k) .
$$

Then

$$
\begin{aligned}
& \beta^{-1}\left(x^{(1)}(k)-k x^{(0)}(1)\right)=F(k), \\
& x^{(1)}(k)=\beta F(k)+k x^{(0)}(1), \\
& x^{(1)}(k+1)=\beta F(k+1)+(k+1) x^{(0)}(1) .
\end{aligned}
$$

\section{Inertia Nonhomogenous Discrete Gray Model (INDGM)}

The gray model is one of the core components of gray system theory. It is characterized by its simple calculation, which is superior to traditional prediction methods. The nonhomogenous discrete gray model (NDGM) is constructed based on the approximate nonhomogenous index trend. This section introduces the relevant information about the NDGM and inertia nonhomogenous discrete gray model.

3.1. Nonhomogenous Discrete Gray Model (NDGM). Assume that the sequence

$$
X^{(0)}=\left(x^{(0)}(1), x^{(0)}(2), \ldots, x^{(0)}(n)\right)
$$

is an original data sequence. $X^{(1)}$ is an 1-AGO sequence,

$$
X^{(1)}=\left(x^{(1)}(1), x^{(1)}(2), \ldots, x^{(1)}(n)\right),
$$

where $x^{(1)}(k)=\sum_{i=1}^{k} x^{(0)}(i), k=1,2, \ldots, n$.

Definition 9. Assuming that sequences $X^{(0)}$ and $X^{(1)}$ are defined as in (15) and (16), respectively, the equations

$$
\begin{aligned}
x^{(1)}(k+1) & =\beta_{1} x^{(1)}(k)+\beta_{2} k+\beta_{3} \\
\widehat{x}^{(1)}(1) & =x^{(0)}(1)+\beta_{4}
\end{aligned}
$$

define the first-order gray system-prediction model including a variable, referred to as the $\operatorname{NDGM}(1,1)$ [34]. Then the recurrence function is defined as follows:

$$
\begin{aligned}
& \hat{x}^{(1)}(k+1)=\beta_{1}^{k} \widehat{x}^{(1)}(1)+\beta_{2} \sum_{j=1}^{k} j \beta_{1}^{k-j}+\frac{1-\beta_{1}^{k}}{1-\beta_{1}} \beta_{3}, \quad k=1,2, \ldots, n-1, \\
& \hat{x}^{(0)}(k+1)=\hat{x}^{(1)}(k+1)-\widehat{x}^{(1)}(k),
\end{aligned}
$$




$$
\beta_{4}=\frac{\sum_{k=1}^{n-1}\left[x^{(1)}(k+1)-\beta_{1}^{k} x^{(1)}(1)-\beta_{2} \sum_{j=1}^{k} j \beta_{1}^{k-j}-\left(\left(1-\beta_{1}^{k}\right) /\left(1-\beta_{1}\right)\right) \beta_{3}\right] \beta_{1}^{k}}{1+\sum_{k=1}^{n-1}\left(\beta_{1}^{k}\right)^{2}} .
$$

3.2. Parameter Space of the NDGM. Assuming that sequences $X^{(0)}$ and $X^{(1)}$ are defined as in (15) and (16), respectively, the equation

$$
\alpha(k)=(\alpha(2), \alpha(3), \ldots, \alpha(n))
$$

is called the acceleration sequence of the original sequence, where

$$
\alpha(k)=x^{(0)}(k)-x^{(0)}(k-1) .
$$

Then

$$
f(k)=(f(2), f(3), \ldots, f(n))
$$

is called the external force sequence of the original sequence, where $f(k)=\beta^{-1} \alpha(k)$. Let

$$
\begin{aligned}
& \Phi(k)=\sum_{i=2}^{k} f(i), \\
& F(k)=\sum_{i=2}^{k} \Phi(i), \\
& F(1)=0,
\end{aligned}
$$

where $\Phi(k)$ and $F(k)$ are, respectively, first-order external force combination and second-order external force combination of $f$. Force decompositions of $x^{(1)}(k), x^{(1)}(k+1)$ are as follows:

$$
\begin{aligned}
x^{(1)}(k) & =k x^{(0)}(1)+\beta F(k), \\
x^{(1)}(k+1) & =(k+1) x^{(0)}(1)+\beta F(k+1) .
\end{aligned}
$$

Definition 10. The NDGM can be defined by Definition 9 . Then one has the following:

(1) $\left(\beta_{1}, \beta_{2}, \beta_{3}\right)$ is called the principle parameter space of NDGM, $\beta_{1}, \beta_{2}, \beta_{3}$ are called principle parameters, and $\left(\beta_{1}, \beta_{2}, \beta_{3}\right)$ is the I-order parameter packet of the NDGM.

(2) Let $\beta_{1}=\Delta_{\beta_{1}} / \Delta, \beta_{2}=\Delta_{\beta_{2}} / \Delta, \beta_{3}=\Delta_{\beta_{3}} / \Delta$, let $\Delta_{\beta_{1}}$, $\Delta_{\beta_{2}}, \Delta_{\beta_{3}}, \Delta$ be structure parameters of $\operatorname{NDGM}(1,1)$, and let $\left(\Delta_{\beta_{1}}, \Delta_{\beta_{2}}, \Delta_{\beta_{3}}, \Delta\right)$ be a structure parameter model or structure model.
(3) Let

$$
\begin{aligned}
\Delta_{\beta_{1}}= & \left(F N-G^{2}\right) H+(E G-D N) I \\
& +(D G-E F) M, \\
\Delta_{\beta_{2}}= & (E G-D N) H+\left(C N-E^{2}\right) I \\
& +(D E-C G) M, \\
\Delta_{\beta_{3}}= & (D G-E F) H+(D E-C E) I+\left(C F-D^{2}\right) M, \\
\Delta= & C F N+2 D G E-C G^{2}-N D^{2}-F E^{2},
\end{aligned}
$$

where

$$
\begin{aligned}
C & =\sum_{k=1}^{n-1} x^{(1)}(k)^{2}, \\
D & =\sum_{k=1}^{n-1} k x^{(1)}(k), \\
E & =\sum_{k=1}^{n-1} x^{(1)}(k), \\
F & =\sum_{k=1}^{n-1} k^{2}, \\
G & =\sum_{k=1}^{n-1} k, \\
H & =\sum_{k=1}^{n-1} x^{(1)}(k) x^{(1)}(k+1), \\
I & =\sum_{k=1}^{n-1} k x^{(1)}(k+1), \\
M & =\sum_{k=1}^{n-1} x^{(1)}(k+1), \\
N & =n-1 .
\end{aligned}
$$

C, $D, E, H, I, M$ are called component parameters of NDGM, $(C, D, E, H, I, M)$ is called the componentparameter space or component-parameter model of NDGM. 
Then, second-order parameter of the componentparameter space $(C, D, E, H, I, M)$ is solved by substituting (23) into $C, D, E, H, I, M$, which yields

$$
\begin{aligned}
C= & \sum_{k=1}^{n-1} x^{(1)}(k)^{2}=\sum_{k=1}^{n-1}\left[k x^{(0)}(1)+\beta F(k)\right]^{2} \\
= & \sum_{k=1}^{n-1} k^{2} x^{(0)}(1)^{2} \\
& +\beta \sum_{k=1}^{n-1} 2 k x^{(0)}(1) F(k)+\beta^{2} \sum_{k=1}^{n-1} F(k)^{2} \\
= & C_{0}+\beta C_{1}+\beta^{2} C_{2},
\end{aligned}
$$

where $C_{0}=\sum_{k=1}^{n-1} k^{2} x^{(0)}(1)^{2}, C_{1}=\sum_{k=1}^{n-1} 2 k x^{(0)}(1) F(k), C_{2}=$ $\sum_{k=1}^{n-1} F(k)^{2}$;

$$
\begin{aligned}
D & =\sum_{k=1}^{n-1} k x^{(1)}(k)=\sum_{k=1}^{n-1} k\left(k x^{(0)}(1)+\beta F(k)\right) \\
& =\sum_{k=1}^{n-1} k^{2} x^{(0)}(1)+\beta \sum_{k=1}^{n-1} k F(k)=D_{0}+\beta D_{1},
\end{aligned}
$$

where $D_{0}=\sum_{k=1}^{n-1} k^{2} x^{(0)}(1), D_{1}=\sum_{k=1}^{n-1} k F(k)$;

$$
E=\sum_{k=1}^{n-1} x^{(1)}(k)=\sum_{k=1}^{n-1} k x^{(0)}(1)+\beta \sum_{k=1}^{n-1} F(k)=E_{0}+\beta E_{1},
$$

where $E_{0}=\sum_{k=1}^{n-1} k x^{(0)}(1), E_{1}=\sum_{k=1}^{n-1} F(k)$,

$$
\begin{aligned}
H & =\sum_{k=1}^{n-1} x^{(1)}(k) x^{(1)}(k+1) \\
& =\sum_{k=2}^{n}\left[k x^{(0)}(1)+\beta(F(k))\right] \\
& \cdot\left[(k+1) x^{(0)}(1)+\beta(F(k+1))\right]=\sum_{k=1}^{n-1} k(k+1) \\
& \cdot x^{(0)}(1)^{2} \\
& +\beta \sum_{k=1}^{n-1}\left[k F(k+1) x^{(0)}(1)+(k+1) F(k) x^{(0)}(1)\right] \\
& +\beta^{2} \sum_{k=1}^{n-1} F(k+1) F(k)=H_{0}+\beta H_{1}+\beta^{2} H_{2},
\end{aligned}
$$

where $H_{0}=\sum_{k=1}^{n-1} k(k+1) x^{(0)}(1)^{2}, H_{1}=\sum_{k=1}^{n-1}[k F(k+$ 1) $\left.x^{(0)}(1)+(k+1) F(k) x^{(0)}(1)\right], H_{2}=\sum_{k=1}^{n-1} F(k+1) F(k)$;

$$
\begin{aligned}
I & =\sum_{k=1}^{n-1} k x^{(1)}(k+1) \\
& =\sum_{k=1}^{n-1} k\left[(k+1) x^{(0)}(1)+\beta F(k+1)\right] \\
& =\sum_{k=1}^{n-1} k(k+1) x^{(0)}(1)+\beta \sum_{k=1}^{n-1} k F(k+1)=I_{0}+\beta I_{1},
\end{aligned}
$$

where $I_{0}=\sum_{k=1}^{n-1} k(k+1) x^{(0)}(1), I_{1}=\sum_{k=1}^{n-1} k F(k+1)$;

$$
\begin{aligned}
M & =\sum_{k=1}^{n-1} x^{(1)}(k+1)=\sum_{k=1}^{n-1}(k+1) x^{(0)}(1)+\beta F(k+1) \\
& =\sum_{k=1}^{n-1}(k+1) x^{(0)}(1)+\beta \sum_{k=1}^{n-1} F(k+1)=M_{0}+\beta M_{1},
\end{aligned}
$$

where $M_{0}=\sum_{k=1}^{n-1}(k+1) x^{(0)}(1), M_{1}=\sum_{k=1}^{n-1} F(k+1)$.

Substituting (23) into (24) for the structural parameters yields

$$
\begin{aligned}
\Delta_{\beta_{1}} & =\left(F N-G^{2}\right) H+(E G-D N) I+(D G-E F) M \\
& =\left(F N-G^{2}\right)\left(H_{0}+\beta H_{1}+\beta^{2} H_{2}\right) \\
& +\left[\left(E_{0}+\beta E_{1}\right) G-\left(D_{0}+\beta D_{1}\right) N\right]\left(I_{0}+\beta I_{1}\right) \\
& +\left[\left(D_{0}+\beta D_{1}\right) G-\left(E_{0}+\beta E_{1}\right) F\right]\left(M_{0}+\beta M_{1}\right) \\
& =\left(F N-G^{2}\right) H_{0}+\left(E_{0} G-D_{0} N\right) I_{0}+\left(D_{0} G\right. \\
& \left.-E_{0} F\right) M_{0}+\beta\left[\left(F N-G^{2}\right) H_{1}+\left(E_{0} G-D_{0} N\right) I_{1}\right. \\
& \left.+\left(D_{0} G-E_{0} F\right) M_{1}+\left(D_{1} G-E_{1} F\right) M_{0}\right] \\
& +\beta^{2}\left[\left(F N-G^{2}\right) H_{2}+\left(E_{1} G-D_{1} N\right) I_{1}\right. \\
& \left.+\left(D_{1} G-E_{1} F\right) M_{1}\right]=\Delta_{\beta_{10}}+\beta \Delta_{\beta_{11}}+\beta^{2} \Delta_{\beta_{12}} .
\end{aligned}
$$

Similarly,

$$
\begin{aligned}
\Delta_{\beta_{2}} & =\left(E_{0} G-D_{0} N\right) H_{0}-\left(C_{0} N-E_{0}^{2}\right) I_{0}+\left(D_{0} E_{0}\right. \\
& \left.-C_{0} G\right) M_{0}+\beta\left[\left(E_{1} G-D_{1} N\right) H_{0}\right. \\
& +\left(E_{0} G-D_{0} N\right) H_{1}+\left(C_{0} N-E_{0}^{2}\right) I_{1} \\
& +\left(C_{1} N-2 E_{0} E_{1}\right) I_{0}+\left(D_{0} E_{0}-C_{0} G\right) M_{1} \\
& \left.+\left(D_{1} E_{0}+D_{0} E_{1}-C_{1} G\right) M_{0}\right] \\
& +\beta^{2}\left[\left(E_{0} G-D_{0} N\right) H_{2}+\left(E_{1} G-D_{1} N\right) H_{1}\right. \\
& +\left(C_{1} N-2 E_{0} E_{1}\right) I_{1}+\left(C_{2} N-E_{1}^{2}\right) I_{0}
\end{aligned}
$$




$$
\begin{aligned}
+ & \left(D_{1} E_{1}-C_{2} G\right) M_{0} \\
+ & \left.\left(D_{0} E_{1}+D_{1} E_{0}-C_{1} G\right) M_{1}\right] \\
+ & \beta^{3}\left[\left(E_{1} G-D_{1} N\right) H_{2}+\left(C_{2} N-E_{1}^{2}\right) I_{1}\right. \\
+ & \left.\left(D_{1} E_{1}-C_{2} G\right) M_{1}\right]=\Delta_{\beta_{20}}+\beta \Delta_{\beta_{21}}+\beta^{2} \Delta_{\beta_{22}} \\
+ & \beta^{3} \Delta_{\beta_{23}} ; \\
\Delta_{\beta_{3}} & =\left(D_{0} G-E_{0} F\right) H_{0}+\left(D_{0} E_{0}-C_{0} G\right) I_{0}+\left(C_{0} F\right. \\
& \left.-D_{0}^{2}\right) M_{0}+\beta\left[\left(D_{0} G-E_{0} F\right) H_{1}\right. \\
+ & \left(D_{1} G-E_{1} F\right) H_{0}+\left(D_{1} E_{0}+D_{0} E_{1}-C_{1} G\right) I_{0} \\
+ & \left(D_{0} E_{0}-C_{0} E_{0}\right) I_{1}+\left(C_{0} F-D_{0}^{2}\right) M_{1} \\
+ & \left.\left(C_{1} F-2 D_{0} D_{1}\right) M_{0}\right]+\beta^{2}\left[\left(D_{0} G-E_{0} F\right) H_{2}\right. \\
+ & \left(D_{1} G-E_{1} F\right) H_{1}+\left(D_{1} E_{0}+D_{0} E_{1}-C_{1} G\right) I_{1} \\
+ & \left(D_{1} E_{1}-C_{2} G\right) I_{0}+\left(C_{2} F-D_{1}^{2}\right) M_{0} \\
+ & \left.\left(C_{1} F-2 D_{0} D_{1}\right) M_{1}\right]+\beta^{3}\left[\left(D_{1} G-E_{1} F\right) H_{2}\right. \\
& \left.+\left(D_{1} E_{1}-C_{2} G\right) I_{1}+\left(C_{2} F-D_{1}^{2}\right) M_{1}\right]=\Delta_{\beta_{30}} \\
+ & \beta \Delta_{\beta_{31}}+\beta^{2} \Delta_{\beta_{32}}+\beta^{3} \Delta_{\beta_{33}} ; \\
& +C_{0} F N+2 D_{0} E_{0} G-C_{0} G^{2}-D_{0}^{2} N-E_{0}^{2} F \\
& \left.C_{2} G^{2}-D_{1}^{2} N-E_{1}^{2} F\right)=\Delta_{0}+\beta D_{1}+\beta^{2} \Delta_{2} .
\end{aligned}
$$

3.3. Inertia Nonhomogenous Discrete Gray Model (INDGM). Assuming that sequence $X^{(0)}$ is defined by (15), the acceleration sequence is defined as follows:

$$
\alpha(k)=x^{(0)}(k)-x^{(0)}(k-1),
$$

and the acceleration sequence can be represented as

$$
\alpha(k)=(\alpha(2), \alpha(3), \ldots, \alpha(n)) ;
$$

$f(k)$ is the external force of original sequence $X^{(0)}$ on point $k$. Let $f(k)=\beta^{-1} \alpha(k)$.

The incentive coefficient is $\beta$, and the inertia coefficient is $\beta^{-1}$. At the same time,

$$
\begin{aligned}
f(k) & =\left(\beta^{-1} \alpha(2), \beta^{-1} \alpha(3), \ldots, \beta^{-1} \alpha(n)\right) \\
& =(f(2), f(3), \ldots, f(n)) .
\end{aligned}
$$

Based on (32)-(35), Definition 11 can be given.
Definition 11. Let

$$
\begin{aligned}
& \left(\beta_{1}, \beta_{2}, \beta_{3}\right) \\
& \quad=\left(\beta_{1}\left(\beta, \beta^{2}\right), \beta_{2}\left(\beta, \beta^{2}, \beta^{3}\right), \beta_{3}\left(\beta, \beta^{2}, \beta^{3}\right)\right),
\end{aligned}
$$

where

$$
\begin{aligned}
& \beta_{1}=\frac{\Delta_{\beta_{10}}+\beta \Delta_{\beta_{11}}+\beta^{2} \Delta_{\beta_{12}}}{\Delta_{0}+\beta \Delta_{1}+\beta^{2} \Delta_{2}}, \\
& \beta_{2}=\frac{\Delta_{\beta_{20}}+\beta \Delta_{\beta_{21}}+\beta^{2} \Delta_{\beta_{22}}+\beta^{3} \Delta_{\beta_{23}}}{\Delta_{0}+\beta \Delta_{1}+\beta^{2} \Delta_{2}}, \\
& \beta_{3}=\frac{\Delta_{\beta_{30}}+\beta \Delta_{\beta_{31}}+\beta^{2} \Delta_{\beta_{32}}+\beta^{3} \Delta_{\beta_{33}}}{\Delta_{0}+\beta \Delta_{1}+\beta^{2} \Delta_{2}},
\end{aligned}
$$

with

$$
\begin{aligned}
\Delta_{0}= & C_{0} F N+2 D_{0} E_{0} G-C_{0} G^{2}-D_{0}^{2} N-E_{0}^{2} F, \\
\Delta_{1} & \\
= & C_{1} F N+2 D_{0} E_{1} G+2 D_{1} E_{0} G-C_{1} G^{2} \\
& -2 D_{0} D_{1} N-2 E_{0} E_{1} F, \\
\Delta_{2}= & C_{2} F N+2 D_{1} E_{1} G-C_{2} G^{2}-D_{1}^{2} N-E_{1}^{2} F, \\
\Delta_{\beta_{10}} & \\
= & \left(F N-G^{2}\right) H_{0}+\left(E_{0} G-D_{0} N\right) I_{0} \\
& +\left(D_{0} G-E_{0} F\right) M_{0}, \\
\Delta_{\beta_{11}} & \\
= & \left(F N-G^{2}\right) H_{1}+\left(E_{1} G-D_{1} N\right) I_{0} \\
& +\left(E_{0} G-D_{0} N\right) I_{1}+\left(D_{0} G-E_{0} F\right) M_{1} \\
& +\left(D_{1} G-E_{1} F\right) M_{0},
\end{aligned}
$$

$\Delta_{\beta_{12}}$

$$
=\left(F N-G^{2}\right) H_{2}+\left(E_{1} G-D_{1} N\right) I_{1}
$$$$
+\left(D_{1} G-E_{1} F\right) M_{1},
$$

$\Delta_{\beta_{20}}$

$$
\begin{aligned}
= & E_{0} H_{0} G-D_{0} H_{0} N-C_{0} N I_{0}+E_{0}^{2} I_{0}+D_{0} E_{0} M_{0} \\
& -C_{0} M_{0} G,
\end{aligned}
$$

$\Delta_{\beta_{21}}$

$$
\begin{aligned}
= & \left(E_{1} G H_{0}-D_{0} N H_{0}+E_{0} H_{1} G-D_{0} H_{1} N\right) \\
& -\left(C_{0} N I_{1}-E_{0}^{2} I_{1}-C_{1} N I_{0}-2 E_{0} E_{1} I_{0}\right) \\
& +\left(D_{0} E_{0} M_{1}-C_{0} M_{1} G+D_{1} E_{0} M_{0}-C_{1} M_{0} G\right),
\end{aligned}
$$


$\Delta_{\beta_{22}}$

$$
\begin{aligned}
= & \left(E_{0} G H_{2}-D_{0} N H_{2}+E_{1} G H_{1}-D_{1} N H_{1}\right) \\
& -\left(C_{1} N I_{1}-2 E_{0} E_{1} I_{1}-C_{2} N I_{0}-E_{1}^{2} I_{0}\right) \\
& +\left(D_{1} E_{1} M_{0}-C_{1} M_{1} G+D_{1} E_{1} M_{0}-C_{1} M_{1} G\right), \\
\Delta_{\beta_{23}} & =E_{1} G H_{2}-D_{1} N H_{2}-C_{2} N I_{1}-E_{1}^{2} I_{1}, \\
\Delta_{\beta_{30}} & \\
= & \left(D_{0} G-E_{0} F\right) H_{0}+\left(D_{0} E_{0}-C_{0} G\right) I_{0} \\
& +\left(C_{0} F-D_{0}^{2}\right) M_{0},
\end{aligned}
$$

$\Delta_{\beta_{31}}$

$$
\begin{aligned}
= & \left(D_{0} G-E_{0} F\right) H_{1}+\left(D_{1} G-E_{1} F\right) H_{0} \\
& +\left(D_{0} E_{0}-C_{0} G\right) I_{1}+\left(D_{1} E_{0}+D_{0} E_{1}-C_{1} G\right) I_{0} \\
& +\left(C_{0} F-D_{0}^{2}\right) M_{1}+\left(C_{1} F-2 D_{0} D_{1}\right) M_{0},
\end{aligned}
$$

$\Delta_{\beta_{32}}$

$$
\begin{aligned}
= & \left(D_{0} G-E_{0} F\right) H_{2}+\left(D_{1} G-E_{1} F\right) H_{1} \\
& +\left(D_{1} E_{0}+D_{0} E_{1}-C_{1} G\right) I_{1}+\left(D_{1} E_{1}-C_{2} G\right) I_{0} \\
& +\left(C_{2} F-D_{1}^{2}\right) M_{0}+\left(C_{1} F-2 D_{0} D_{1}\right) M_{1},
\end{aligned}
$$

$\Delta_{\beta_{33}}$

$$
\begin{aligned}
= & \left(D_{1} G-E_{1} F\right) H_{2}+\left(D_{1} E_{1}-C_{2} G\right) I_{1} \\
& +\left(C_{2} F-D_{1}^{2}\right) M_{1} .
\end{aligned}
$$

Then

(1) $\left(\beta_{1}, \beta_{2}, \beta_{3}\right)=\left(\beta_{10}, \beta_{20}, \beta_{30}\right)=\left(\Delta_{\beta_{10}} / \Delta_{0}, \Delta_{\beta_{20}} / \Delta_{0}\right.$, $\left.\Delta_{\beta_{30}} / \Delta_{0}\right)$ is called the competency model of NDGM;

(2) $\left(\beta_{11}, \beta_{21}, \beta_{31}\right)=\left(\beta_{1}(\beta), \beta_{2}(\beta), \beta_{3}(\beta)\right)=\left(\left(\Delta_{\beta_{10}}+\right.\right.$ $\left.\beta \Delta_{\beta_{11}}\right) /\left(\Delta_{0}+\beta \Delta_{1}\right),\left(\Delta_{\beta_{20}}+\beta \Delta_{\beta_{21}}\right) /\left(\Delta_{0}+\beta \Delta_{1}\right),\left(\Delta_{\beta_{30}}+\right.$ $\left.\left.\beta \Delta_{\beta_{31}}\right) /\left(\Delta_{0}+\beta \Delta_{1}\right)\right)$ is called the first-order inertia (incentive) model of NDGM, denoted as the FINDGM;

(3) $\left(\beta_{12}, \beta_{22}, \beta_{32}\right)=\left(\beta_{1}\left(\beta^{2}\right), \beta_{2}\left(\beta^{2}\right), \beta_{3}\left(\beta^{2}\right)\right)=\left(\left(\Delta_{\beta_{10}}+\right.\right.$ $\left.\beta \Delta_{\beta_{11}}+\beta^{2} \Delta_{\beta_{12}}\right) /\left(\Delta_{0}+\beta \Delta_{1}+\beta^{2} \Delta_{2}\right),\left(\Delta_{\beta_{20}}+\right.$ $\left.\beta \Delta_{\beta_{21}}+\beta^{2} \Delta_{\beta_{22}}\right) /\left(\Delta_{0}+\beta \Delta_{1}+\beta^{2} \Delta_{2}\right),\left(\Delta_{\beta_{30}}+\beta \Delta_{\beta_{31}}+\right.$ $\left.\left.\beta^{2} \Delta_{\beta_{32}}\right) /\left(\Delta_{0}+\beta \Delta_{1}+\beta^{2} \Delta_{2}\right)\right)$ is called the second-order inertia (incentive) model of NDGM, denoted as the SINDGM;

(4) $\left(\beta_{13}, \beta_{23}, \beta_{33}\right)=\left(\beta_{1}\left(\beta^{3}\right), \beta_{2}\left(\beta^{3}\right), \beta_{3}\left(\beta^{3}\right)\right)=\left(\left(\Delta_{\beta_{10}}+\right.\right.$ $\left.\beta \Delta_{\beta_{11}}+\beta^{2} \Delta_{\beta_{12}}\right) /\left(\Delta_{0}+\beta \Delta_{1}+\beta^{2} \Delta_{2}\right),\left(\Delta_{\beta_{20}}+\beta \Delta_{\beta_{21}}+\right.$ $\left.\beta^{2} \Delta_{\beta_{22}}+\beta^{3} \Delta_{\beta_{23}}\right) /\left(\Delta_{0}+\beta \Delta_{1}+\beta^{2} \Delta_{2}\right),\left(\Delta_{\beta_{30}}+\beta \Delta_{\beta_{31}}+\right.$ $\left.\left.\beta^{2} \Delta_{\beta_{32}}+\beta^{3} \Delta_{\beta_{33}}\right) /\left(\Delta_{0}+\beta \Delta_{1}+\beta^{2} \Delta_{2}\right)\right)$ is called the third-order inertia (incentive) model of NDGM, denoted as the TINDGM.

\subsection{Properties of the INDGM}

Theorem 12. The competency model and the FINDGM of the NDGM inertia model do not exist.

Proof. Definition 11 and (29)-(34) can be substituted into the expressions for $\Delta_{0}$ and $\Delta_{1}$. Then $\Delta_{0}=0, \Delta_{1}=0$. From Definition 11, parts (1) and (2), it is clear that the competency model and the first-order inertia model of the NDGM inertia model do not exist.

Theorem 13. The TINDGM is equivalent to the NDGM.

Proof. From the definition of the structure parameters of the NDGM,

$$
\begin{aligned}
& \beta_{1}=\frac{\Delta_{\beta_{1}}}{\Delta}, \\
& \beta_{2}=\frac{\Delta_{\beta_{2}}}{\Delta}, \\
& \beta_{3}=\frac{\Delta_{\beta_{3}}}{\Delta} .
\end{aligned}
$$

From (32)-(35), the TINDGM is equivalent to the NDGM, which is equivalent to the NDGM. Thus, TINDGM is equivalent to the NDGM.

From Definition 11, the four INDGM are named according to the magnitude of the exponent $\beta$ of the inertial coefficient. The exponent of the inertia coefficient $\beta$ is closely related to the mechanical decomposition of the data. Meanwhile, from Theorem 12, the competency model and the FINDGM of the inertia NDGM do not exist, and from Theorem 13, the TINDGM is equivalent to the NDGM. Thus, from the point of view of the mechanical decomposition of the data, the classical NDGM is an evolutionary process with four inertial models, from the competency model, the FINDGM, and the SIDGM to the TINDGM; however, the FINDGM and SINDGM do not exist, which implies that there does not exist a mechanical decomposition like this for the NDGM. At the same time, the modeling mechanism of the NDGM is obtained according to the data mechanical-decomposition process of the exponent of the inertia coefficient $\beta$ from low to high.

Theorem 14. The restored value of the INDGM is

$$
\widehat{x}^{(0)}(k+1)=\widehat{x}^{(1)}(k+1)-\widehat{x}^{(1)}(k),
$$

where 


$$
\begin{aligned}
\widehat{x}^{(1)}(k+1) & =\beta_{1 i}^{k} \widehat{x}^{(1)}(1)+\beta_{2 i} \sum_{j=1}^{k} j \beta_{1 i}^{k-j}+\frac{1-\beta_{1 i}^{k}}{1-\beta_{1 i}} \beta_{3 i}, \quad i=0,1,2,3, \quad k=1,2, \ldots, n-1, \\
\beta_{4 i} & =\frac{\sum_{k=1}^{n-1}\left[x^{(1)}(k+1)-\beta_{1 i}^{k} x^{(1)}(1)-\beta_{2 i} \sum_{j=1}^{k} j \beta_{1}^{k-j}-\left(\left(1-\beta_{1 i}^{k}\right) /\left(1-\beta_{1 i}\right)\right) \beta_{3 i}\right] \beta_{1 i}^{k}}{1+\sum_{k=1}^{n-1}\left(\beta_{1 i}^{k}\right)^{2}}, \quad i=0,1,2,3 .
\end{aligned}
$$

The same sequence $X^{(0)}$ has different meanings under different thoughts and processes of things. At the same time, the data have different mechanical decompositions, depending on the data source. Next, we examine whether changes in the inertia coefficient $\beta$ for the same data affect the accuracy of the model.

Theorem 15. For the same sequence $X^{(0)}$ and different decompositions of the data, the second-order parameters of the component parameters $C, D, E, H, I, M$ and structure parameters $\left(\Delta_{\beta_{1}}, \Delta_{\beta_{2}}, \Delta_{\beta_{3}}, \Delta\right)$ of the model will change, but the values of the model parameters $\left(\beta_{1 i}, \beta_{2 i}, \beta_{3 i}\right), i=0,1,2,3$ are invariant; that is, the simulation accuracy of the model is not influenced by the choice of decomposition.

Proof. Assume that sequence $X^{(0)}$ has an incentive coefficient of the form $\gamma=\sigma \beta$. Then under two different incentive coefficients,

$$
\begin{aligned}
\alpha(k) & =\alpha_{\gamma}(k)=\beta_{1} f_{\gamma}(k)=\sigma \beta f_{\gamma}(k)=\alpha_{\beta}(k) \\
& =\beta f_{\beta}(k) .
\end{aligned}
$$

Therefore, the force element $f$ satisfies $f_{\beta}(k)=\sigma f_{\gamma}(k)$,

$$
\Phi_{\beta}(k)=\sum_{i=2}^{k} f_{\beta}(i)=\sum_{i=2}^{k} \sigma f_{\gamma}(i)=\sigma \Phi_{\gamma}(k) .
$$

Thus, the first-order combination of the force element satisfies $(1 / \sigma) \Phi_{\beta}(k)=\Phi_{\gamma}(k)$; and

$$
F_{\beta}(k)=\sum_{i=2}^{k} \Phi_{\beta}(i)=\sum_{i=2}^{k} \sigma \Phi_{\gamma}(i)=\sigma F_{\gamma}(k),
$$

It follows that the second-order combination of the force element satisfies $(1 / \sigma) F_{\beta}(k)=F_{\gamma}(k)$.

Now, assuming that the component parameters of inertia exponent $\beta$ are

$$
C_{\beta}, D_{\beta}, E_{\beta}, H_{\beta}, I_{\beta}, M_{\beta} \text {, }
$$

the component parameters of inertia exponent $\gamma$ are $C_{\gamma}, D_{\gamma}, E_{\gamma}, H_{\gamma}, I_{\gamma}, M_{\gamma}$, where $\gamma=\sigma \beta$. From (26),

$$
\begin{aligned}
C_{\beta}= & \sum_{k=1}^{n-1} k^{2} x^{(0)}(1)^{2} \\
& +\beta \sum_{k=1}^{n-1} 2 k x^{(0)}(1) F_{\beta}(k)+\beta^{2} \sum_{k=1}^{n-1} F_{\beta}(k)^{2} \\
= & C_{\beta 0}+\beta C_{\beta 1}+\beta^{2} C_{\beta 2}, \\
C_{\gamma}= & \sum_{k=1}^{n-1} k^{2} x^{(0)}(1)^{2} \\
& +\gamma \sum_{k=1}^{n-1} 2 k x^{(0)}(1) F_{\gamma}(k)+\gamma^{2} \sum_{k=1}^{n-1} F_{\gamma}(k)^{2} \\
= & C_{\gamma 0}+\gamma C_{\gamma 1}+\gamma^{2} C_{\gamma 2} .
\end{aligned}
$$

Comparing (50) with (51), (48) implies

$$
\begin{aligned}
& C_{\beta 0}=C_{\gamma 0}, \\
& C_{\beta 1}=\sigma C_{\gamma 1}, \\
& C_{\beta 2}=\sigma^{2} C_{\gamma 2} .
\end{aligned}
$$

Similarly,

$$
\begin{aligned}
& C_{\beta 0}=C_{\gamma 0}, \\
& C_{\beta 1}=\sigma C_{\gamma 1}, \\
& C_{\beta 2}=\sigma^{2} C_{\gamma 2}, \\
& D_{\beta 0}=D_{\gamma 0}, \\
& D_{\beta 1}=\sigma D_{\gamma 1}, \\
& E_{\beta 0}=E_{\gamma 0}, \\
& E_{\beta 1}=\sigma E_{\gamma 1}, \\
& H_{\beta 0}=H_{\gamma 0}, \\
& H_{\beta 1}=\sigma H_{\gamma 1}, \\
& H_{\beta 2}=\sigma^{2} H_{\gamma 2},
\end{aligned}
$$




$$
\begin{gathered}
I_{\beta 0}=I_{\gamma 0}, \\
I_{\beta 1}=\sigma I_{\gamma 1}, \\
M_{\beta 0}=M_{\gamma 0}, \\
M_{\beta 1}=\sigma M_{\gamma 1} .
\end{gathered}
$$

By (51), we have

$$
\begin{aligned}
\Delta_{0}= & C_{\beta 0} F N+2 D_{\beta 0} E_{\beta 0} G-C_{\beta 0} G^{2}-D_{\beta 0}^{2} N-E_{\beta 0}^{2} F \\
= & C_{\beta 0} F N+2 D_{\beta 0} E_{\beta 0} G-C_{\beta 0} G^{2}-D_{\beta 0}^{2} N-E_{\beta 0}^{2} F \\
= & \Delta_{0}^{\prime}, \\
\Delta_{1}= & C_{\beta 1} F N+2 D_{\beta 0} E_{\beta 1} G+2 D_{\beta 1} E_{\beta 0} G-C_{\beta 1} G^{2} \\
& -2 D_{\beta 0} D_{\beta 1} N-2 E_{\beta 0} E_{\beta 1} F \\
= & \sigma C_{\gamma 1} F N+2 \sigma D_{\gamma 0} E_{\gamma 1} G+2 \sigma D_{\gamma 1} E_{\gamma 0} G \\
& -\sigma C_{\gamma 1} G^{2}-2 \sigma D_{\gamma 0} D_{\gamma 1} N-2 \sigma E_{\gamma 0} E_{\gamma 1} F=\sigma \Delta_{1}^{\prime}, \\
\Delta_{2}= & C_{\beta 2} F N+2 D_{\beta 1} E_{\beta 1} G-C_{\beta 2} G^{2}-D_{\beta 1}^{2} N-E_{\beta 1}^{2} F \\
= & \sigma^{2} C_{\gamma 2} F N+2 \sigma^{2} D_{\gamma 1} E_{\gamma 1} G-\sigma^{2} C_{\gamma 2} G^{2}-\sigma^{2} D_{\gamma 1}^{2} N \\
& -\sigma^{2} E_{\gamma 1}^{2} F=\sigma^{2} \Delta_{2}^{\prime} .
\end{aligned}
$$

Similarly,

$$
\begin{aligned}
& \Delta_{\beta_{10}}=\Delta_{\gamma_{10}}, \\
& \Delta_{\beta_{11}}=\sigma \Delta_{\gamma_{11}}, \\
& \Delta_{\beta_{12}}=\sigma^{2} \Delta_{\gamma_{12}}, \\
& \Delta_{\beta_{20}}=\Delta_{\gamma_{20}}, \\
& \Delta_{\beta_{21}}=\sigma \Delta_{\gamma_{21}}, \\
& \Delta_{\beta_{22}}=\sigma^{2} \Delta_{\gamma_{22}}, \\
& \Delta_{\beta_{23}}=\sigma^{3} \Delta_{\gamma_{23}}, \\
& \Delta_{\beta_{30}}=\Delta_{\gamma_{30}}, \\
& \Delta_{\beta_{31}}=\sigma \Delta_{\gamma_{31}}, \\
& \Delta_{\beta_{32}}=\sigma^{2} \Delta_{\gamma_{32}}, \\
& \Delta_{\beta_{33}}=\sigma^{3} \Delta_{\gamma_{33}}, \\
& \Delta_{\beta_{34}}=\sigma^{4} \Delta_{\gamma_{34}} .
\end{aligned}
$$

(1) Competency model $\left(\beta_{10}, \beta_{20}, \beta_{30}\right)=\left(\Delta_{\beta_{11}} / \Delta_{0}, \Delta_{\beta_{20}} /\right.$ $\left.\Delta_{0}, \Delta_{\beta_{30}} / \Delta_{0}\right)$ does not include $\beta$, so $\left(\beta_{1}, \beta_{2}, \beta_{3}\right)$ does not change with changes in $\beta$.
(2) The FNDGM is $\left(\beta_{11}, \beta_{21}, \beta_{31}\right)=\left(\left(\Delta_{\beta_{10}}+\beta \Delta_{\beta_{11}}\right) /\left(\Delta_{0}+\right.\right.$ $\left.\beta \Delta_{1}\right),\left(\Delta_{\beta_{20}}+\beta \Delta_{\beta_{21}}\right) /\left(\Delta_{0}+\beta \Delta_{1}\right),\left(\Delta_{\beta_{30}}+\beta \Delta_{\beta_{31}}\right) /\left(\Delta_{0}+\right.$ $\left.\beta \Delta_{1}\right)$ ), where for $\beta$ and $\gamma, \gamma=\sigma \beta$, substituting (54)(55) into $\beta_{1}^{\prime}=\left(\Delta_{\gamma_{10}}+\gamma \Delta_{\gamma_{11}}\right) /\left(\Delta_{0}^{\prime}+\gamma \Delta_{1}^{\prime}\right)$ yields

$$
\begin{aligned}
\beta_{11}^{\prime} & =\frac{\Delta_{\gamma_{10}}+\gamma \Delta_{\gamma_{11}}}{\Delta_{0}^{\prime}+\gamma \Delta_{1}^{\prime}}=\frac{\Delta_{\beta_{10}}+\sigma \beta\left((1 / \sigma) \Delta_{\beta_{11}}\right)}{\Delta_{0}+\sigma \beta\left((1 / \sigma) \Delta_{1}\right)} \\
& =\frac{\Delta_{\beta_{10}}+\beta \Delta_{\beta_{11}}}{\Delta_{0}+\beta \Delta_{1}}=\beta_{11} .
\end{aligned}
$$

Thus, $\beta_{11}$ does not change with changes in the inertia parameter. Similarly, it can be shown that $\beta_{21}, \beta_{31}$ do not change with changes in the inertia parameter.

By substituting (54)-(59) into the SINDGM and TINDGM, it can be similarly proved that model parameters $\beta_{1 i}, \beta_{2 i}, \beta_{3 i}, i=2,3$, do not change with the model inertia parameter $\beta$. Therefore, the form of the mechanical decomposition of the data does not affect the accuracy of the model.

According to the modeling mechanism of the proposed INDGM, the flow chart of the new model is presented in Figure 1.

\section{Numerical Example of the INDGM}

Short-term traffic prediction is one of the well-developed areas in transportation. The prediction models in traffic as well as other fields are switching towards data intensive artificial intelligence models or expert systems. In this section, taking the data of traffic flow on Whitemud Drive in Canada as the original data, the simulation results of the INDGM are empirically analyzed.

4.1. Traffic-Flow State Division. Traffic-flow characteristics can be described by the traffic-flow status, and different states of traffic flow show different characteristics. Based on the study of the traffic-flow parameter model and traffic-flow characteristics, the traffic-flow states can be divided into freeflow state, congested-flow state, and blocking-flow state. The traffic characteristics of the three traffic states are as follows.

Under the free-flow state, the traffic-flow rate is small, the road on which the vehicle is driving is unaffected or mildly affected by other vehicles, and the vehicle can maintain a high speed.

Under the congested-flow state, the speed of the vehicle is restricted by the front, but the traffic-flow state is relatively stable, and itself has a certain anti-interference ability. The traffic-flow rate in this state can reach the maximum, but when traffic demand continues to increase, traffic flows produce greater volatility and the traffic-flow rate drops significantly.

Under the jam-flow state, the traffic density is high, speed is restricted strictly by the front, vehicle freedom is small, speed stability is poor, and there is greater volatility. When the traffic-flow rate continues to increase, traffic will exhibit a stop-and-go phenomenon. 


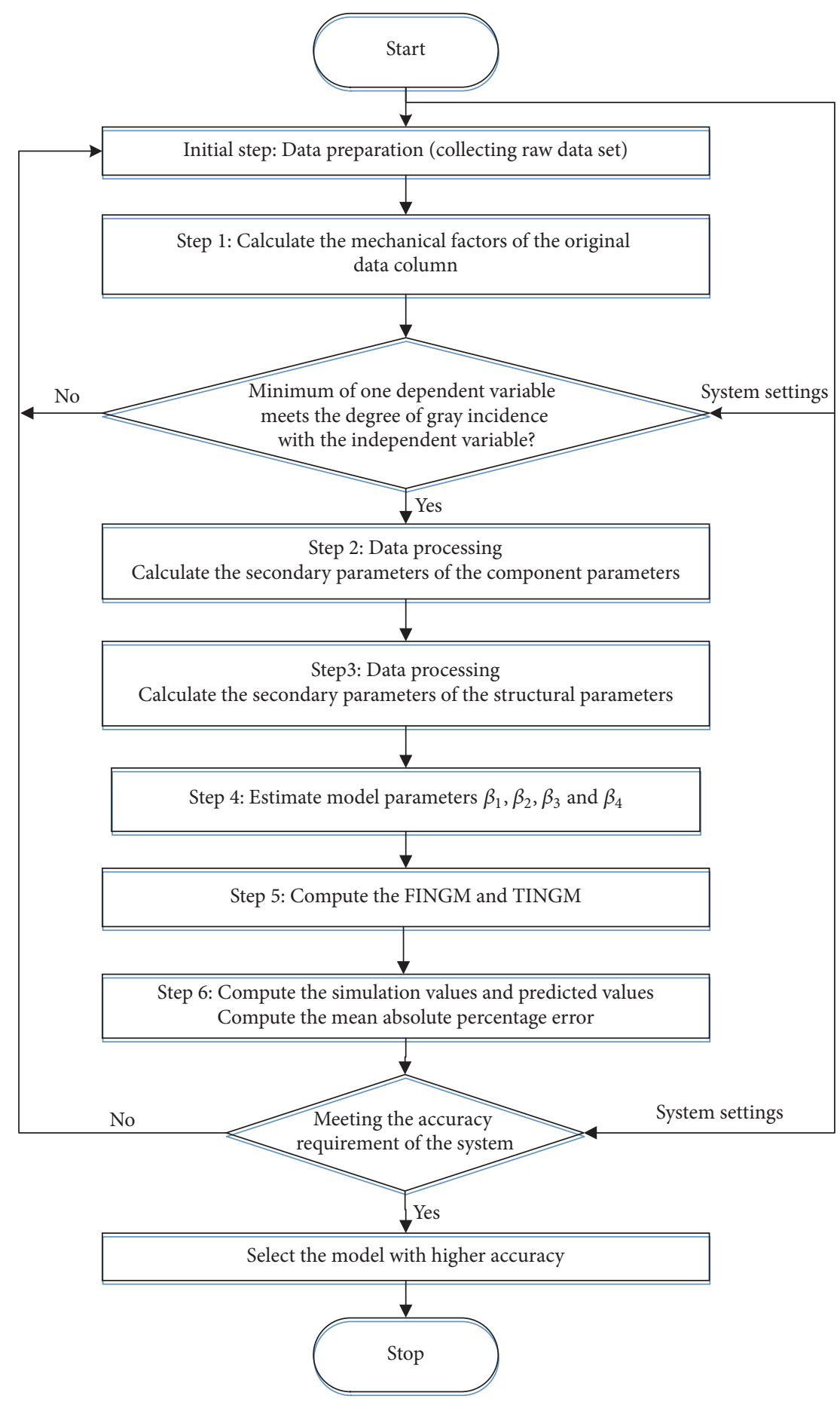

FIgURE 1: The flowchart of the INDGM.

4.2. Establishing the INDGM to Forecast the Amount of Traffic Flow in Canada. The data are taken from the web site http://www.openits.cn/openData/700.jhtml\#xiazai, for the three weeks from August 8, 2015, to August 28, 2015. These data consist of 20-second samples of traffic flow on Whitemud Drive in Canada. This paper uses traffic flow on Sunday, August 23, 12:00-14:00 and 13:50-15:50, and Friday, August 28, 8:50-10:50 and 15:00-17:00. The four stages of data decomposition represent traffic flow on nonworking days and working days, as well as traffic in the free-flow state and congested-flow state. The traffic flow is shown in Table 1.

According to Table 1, the Canadian traffic-flow data show that Canada's traffic flow is generally only in two states, the free-flow state and congested-flow state, and the jamflow state is generally rare. It can be observed that, on the 
TABle 1: Four sections of a road traffic flow in Canada on August 23 and on August 28.

\begin{tabular}{|c|c|c|c|c|}
\hline \multirow{3}{*}{ Serial number } & \multicolumn{2}{|c|}{ August 23, 2015} & \multicolumn{2}{|c|}{ August 28, 2015} \\
\hline & $12: 00-14: 00$ & $13: 50-15: 50$ & $8: 50-10: 50$ & $15: 00-17: 00$ \\
\hline & Free flow & Free flow (steady flow) & Congested flow (smooth traffic) & Congested flow \\
\hline$k=1$ & 114.00 & 114.75 & 135.25 & 159.75 \\
\hline$k=2$ & 116.75 & 112.75 & 134.50 & 171.25 \\
\hline$k=3$ & 119.50 & 115.50 & 121.75 & 178.75 \\
\hline$k=4$ & 126.00 & 131.25 & 134.50 & 176.00 \\
\hline$k=5$ & 129.50 & 112.75 & 139.75 & 180.25 \\
\hline$k=6$ & 125.75 & 123.00 & 137.75 & 184.00 \\
\hline$k=7$ & 120.00 & 116.25 & 121.75 & 207.25 \\
\hline$k=8$ & 131.00 & 117.25 & 134.75 & 185.50 \\
\hline$k=9$ & 127.50 & 117.75 & 138.75 & 208.25 \\
\hline$k=10$ & 117.25 & 113.00 & 140.50 & 221.75 \\
\hline$k=11$ & 127.25 & 125.25 & 140.00 & 190.00 \\
\hline$k=12$ & 114.75 & 109.75 & 137.00 & 186.00 \\
\hline
\end{tabular}

working days, traffic is in the free-flow state and the free flow has large fluctuations and a steady state. On the working day, there is a significant increase in traffic flow compared to the nonworking day, and there is a congested-flow state, in which the congested flow also fluctuates greatly and smoothly.
From Table 1, the first eight data of August 23, 2015, 12:00-14:00, are used for the simulation, while the 4 th data are used as a prediction. The following steps are carried out.

Step 1. Data calculation process: the original data are listed as follows:

$$
\begin{aligned}
X^{(0)} & =(114.00,116.75,119.50,126.00,129.50,125.75,120.00,131.00,127.50,117.25,127.25,114.75), \\
F & =140, \\
G & =28 .
\end{aligned}
$$

Step 2. Calculate the second-order parameters of the component parameters (the values of $\beta_{1 i}, \beta_{2 i}, \beta_{3 i}, i=0,1,2,3$, are independent of $\beta$, so $\beta=1$ ):

$$
\begin{aligned}
& C_{0}=1819440, \\
& C_{1}=216486, \\
& C_{2}=6882.25, \\
& D_{0}=15960, \\
& D_{1}=949.5, \\
& E_{0}=3192, \\
& E_{1}=168, \\
& H_{0}=2183328, \\
& H_{1}=272745, \\
& H_{2}=8924.81, \\
& I_{0}=19152, \\
& I_{1}=1275,
\end{aligned}
$$

$$
\begin{aligned}
& M_{0}=3990 \\
& M_{1}=238.5 .
\end{aligned}
$$

Step 3. Calculate the second-order parameters of the structure parameters $\left(\Delta_{\beta_{1}}, \Delta_{\beta_{2}}, \Delta_{\beta_{3}}, \Delta\right)$ :

$$
\begin{aligned}
\Delta_{2} & =19605.25, \\
\Delta_{\beta_{12}} & =3816.75, \\
\Delta_{\beta_{22}} & =1799889, \\
\Delta_{\beta_{23}} & =186933.47, \\
\Delta_{\beta_{32}} & =2234998.5, \\
\Delta_{\beta_{33}} & =-171357 .
\end{aligned}
$$

Step 4. The values obtained in the second and third steps are substituted into the SIDGM:

$$
\begin{aligned}
& \beta_{1}=0.19468, \\
& \beta_{2}=91.80648,
\end{aligned}
$$


TABLE 2: Comparison of simulative and predictive results between the SINDGM and TINDGM for August 23.

\begin{tabular}{|c|c|c|c|c|c|c|}
\hline Serial number & Free flow & $\begin{array}{c}\text { SINDGM } \\
\text { Simulated data }\end{array}$ & $\begin{array}{c}\text { TINDGM } \\
\text { Simulated data }\end{array}$ & Free flow (steady flow) & $\begin{array}{c}\text { SINDGM } \\
\text { Simulated data }\end{array}$ & $\begin{array}{c}\text { TINDGM } \\
\text { Simulated data }\end{array}$ \\
\hline$k=1$ & 114.00 & 114.00 & 114.00 & 114.75 & 114.75 & 114.75 \\
\hline$k=2$ & 116.75 & 113.17 & 114.57 & 112.75 & 114.82 & 113.84 \\
\hline$k=3$ & 119.50 & 113.84 & 123.65 & 115.50 & 114.72 & 121.70 \\
\hline$k=4$ & 126.00 & 113.97 & 125.41 & 131.25 & 114.76 & 118.81 \\
\hline$k=5$ & 129.50 & 113.98 & 125.76 & 112.75 & 114.75 & 119.87 \\
\hline$k=6$ & 125.75 & 113.99 & 125.82 & 123.00 & 114.75 & 119.48 \\
\hline$k=7$ & 120.00 & 114.00 & 125.83 & 116.25 & 114.74 & 119.63 \\
\hline$k=8$ & 131.00 & 114.00 & 125.84 & 117.25 & 114.75 & 119.57 \\
\hline \multirow[t]{2}{*}{ MAPE } & & 0.0797 & 0.0248 & & 0.0385 & 0.0427 \\
\hline & & Forecasted data & Forecasted data & & Forecasted data & Forecasted data \\
\hline$k=9$ & 127.50 & 114.00 & 125.84 & 117.75 & 114.75 & 119.57 \\
\hline$k=10$ & 117.25 & 114.00 & 125.84 & 113.00 & 114.75 & 119.59 \\
\hline$k=11$ & 127.25 & 114.00 & 125.84 & 125.25 & 114.75 & 119.58 \\
\hline$k=12$ & 114.75 & 114.00 & 125.84 & 109.75 & 114.75 & 119.59 \\
\hline MAPE & & 0.0611 & 0.0485 & & 0.0426 & 0.0522 \\
\hline
\end{tabular}

$$
\begin{aligned}
& \beta_{3}=114, \\
& \beta_{4}=1.0254 .
\end{aligned}
$$

According to the TIDGM model, we have

$$
\begin{aligned}
& \beta_{1}=0.19468, \\
& \beta_{2}=101.34135, \\
& \beta_{3}=105.25964, \\
& \beta_{4}=0.2763 .
\end{aligned}
$$

Step 5. The value of the parameter obtained in Step 4 is substituted into (44):

$$
\begin{aligned}
\widehat{x}^{(1)}(k+1)= & 0.19468^{k} x^{(0)}(1)+91.80648 \sum_{j=1}^{k} j \times 0.19468^{k-j} \\
& +\frac{1-0.19468^{k}}{1-0.19468} \times 114, \\
k=1,2, \ldots, 7, & x^{(0)}(1)=114, x^{(0)}(k)=\hat{x}^{(1)}(k+1)-\hat{x}^{(1)}(k) \\
\widehat{x}^{(1)}(k+1)= & 0.19468^{k} x^{(0)}(1)+101.34135 \sum_{j=1}^{k} j 0.19468^{k-j} \\
& +\frac{1-0.19468^{k}}{1-0.19468} 105.25964, \quad k=1,2, \ldots, n-1 .
\end{aligned}
$$

Step 6. Calculate the simulation values, predictions, and errors.
Substitute the results of Step 5 into (43), and calculate the simulation and predictive values $\hat{x}^{(0)}(k)$ for $x^{(0)}(k)$, which is the original data of Step 1. MAPE is defined as

$$
\text { MAPE }=100 \% \frac{1}{n} \sum_{k=2}^{n}\left|\frac{x^{(0)}(k)-\widehat{x}^{(0)}(k)}{\widehat{x}^{(0)}(k)}\right|,
$$

where $x^{(0)}(k)$ is the original data and $\widehat{x}^{(0)}(k)$ is the simulation and predictive values.

The simulation/prediction values and absolute percentage errors for the SINDGM and TINDGM are shown in Table 2.

According to Table 2, the results of the SINDGM and TINDGM for nonworking days of the two time periods of traffic simulation and prediction are good; the best simulation result is up to 0.0248 , and the best predictive result is up to 0.0426 . Although both are in free-flow states, the amounts of traffic fluctuation are not the same. When the vehicle flow fluctuates greatly, the simulation effect and the prediction effect of the TINDGM are better. When the vehicle flow is relatively stable, the simulation effect and prediction effect of the SINDGM are better than those of the TINDGM. Therefore, when the traffic flow is in the free-flow state and the vehicle flow fluctuates greatly, the TINDGM can be used; when the vehicle flow is relatively stable, the TINDGM can be used. The absolute simulation and prediction percentage errors in Table 2 of the above four models for Canada's traffic flow are illustrated in Figure 2.

Similarly, the above steps are also applied to other sets of data in Table 1 . The simulation/prediction values and absolute percentage errors for the SINDGM and TINDGM are shown in Table 3.

According to Table 3, in the two periods, the vehicle flow is in the congested-flow state on the working day. When the vehicle flow fluctuates less in the congested-flow state, the simulation effect and prediction effect of the SINDGM are better than those of the TINDGM. When the vehicle flow 


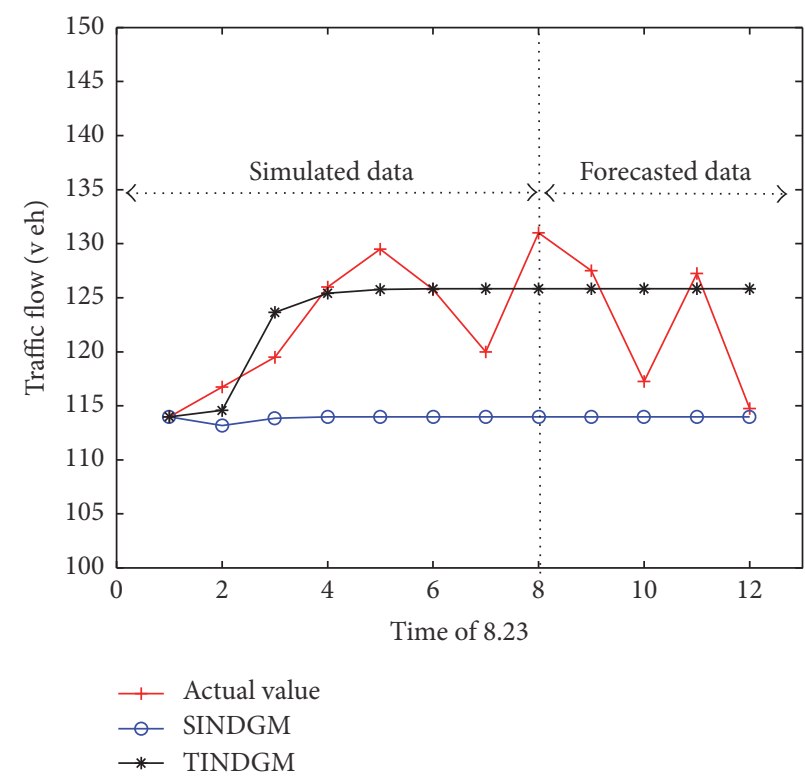

(a) The simulated and forecasted curves of the free flow on August 23

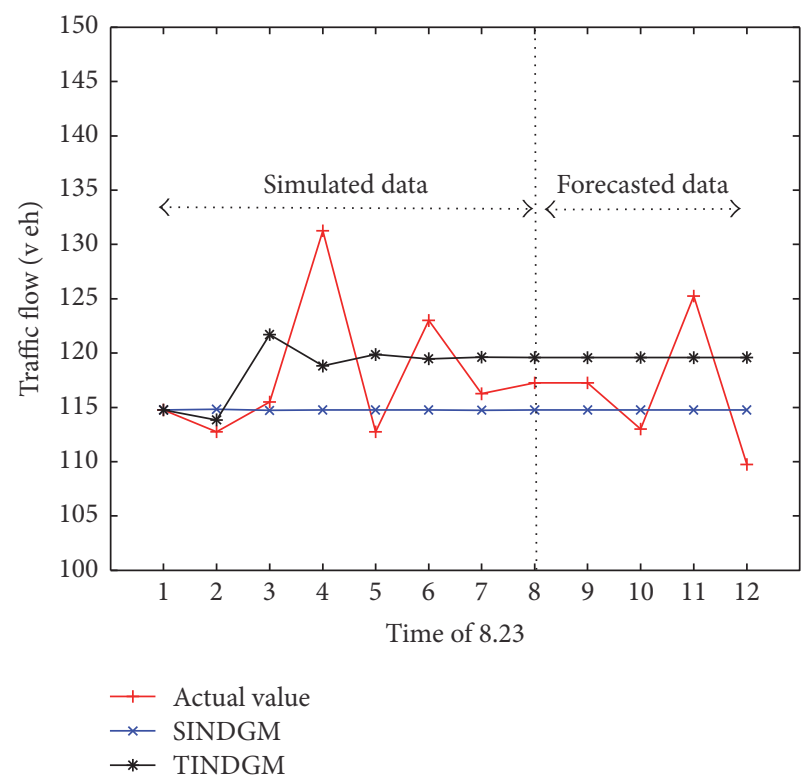

(b) The simulated and forecasted curves of the free flow (steady flow) on August 23

Figure 2

TABLE 3: Comparison of simulative and predictive results between the SINDGM and TINDGM for August 28.

\begin{tabular}{|c|c|c|c|c|c|c|}
\hline $\begin{array}{l}\text { Serial } \\
\text { number }\end{array}$ & Congested flow (smooth traffic) & $\begin{array}{c}\text { SINDGM } \\
\text { Simulated data }\end{array}$ & $\begin{array}{c}\text { TINDGM } \\
\text { Simulated data }\end{array}$ & Congested flow & $\begin{array}{c}\text { SINDGM } \\
\text { Simulated data }\end{array}$ & $\begin{array}{c}\text { TINDGM } \\
\text { Simulated data }\end{array}$ \\
\hline$k=1$ & 135.25 & 135.25 & 135.25 & 159.75 & 159.75 & 159.75 \\
\hline$k=2$ & 134.50 & 135.23 & 129.75 & 171.25 & 144.59 & 167.72 \\
\hline$k=3$ & 121.75 & 135.24 & 132.71 & 178.75 & 149.78 & 176.35 \\
\hline$k=4$ & 134.50 & 135.24 & 132.69 & 176.00 & 153.20 & 182.02 \\
\hline$k=5$ & 139.75 & 135.24 & 132.70 & 180.25 & 155.44 & 185.75 \\
\hline$k=6$ & 137.75 & 135.25 & 132.70 & 184.00 & 156.92 & 188.20 \\
\hline$k=7$ & 121.75 & 135.25 & 132.70 & 207.25 & 157.89 & 189.81 \\
\hline$k=8$ & 134.75 & 135.25 & 132.70 & 185.50 & 158.53 & 190.87 \\
\hline \multirow[t]{2}{*}{ MAPE } & & 0.0409 & 0.0473 & & 0.1594 & 0.0335 \\
\hline & & Forecasted data & Forecasted data & & Forecasted data & Forecasted data \\
\hline$k=9$ & 138.75 & 135.25 & 132.70 & 208.25 & 158.94 & 191.57 \\
\hline$k=10$ & 140.50 & 135.25 & 132.70 & 221.75 & 159.22 & 190.03 \\
\hline$k=11$ & 140.00 & 135.25 & 132.70 & 190.00 & 159.40 & 192.32 \\
\hline$k=12$ & 137.00 & 135.25 & 132.70 & 186.00 & 159.52 & 192.53 \\
\hline MAPE & & 0.0273 & 0.0456 & & 0.2055 & 0.0654 \\
\hline
\end{tabular}

fluctuates greatly, the simulation effect and prediction effect of the SINDGM are better than those of the TINDGM.

Based on the data in Table 3, the absolute simulation and prediction percentage errors of the above two models for Canada's traffic flow are illustrated in Figure 3.

According to Tables 2 and 3 and Figures 2 and 3, general conclusions can be drawn. In the free-flow or congested-flow state, when the vehicle flow is relatively stable, the SINDGM is used to simulate and predict, and when the vehicle flow fluctuates greatly, the TINDGM is used to simulate and predict.

\section{Conclusion}

Short-term traffic-flow forecasting in an intelligent transportation system is an important research area. Short-term traffic-flow research results can provide traffic information for the intelligent transportation system. Such results can also be directly applied to the advanced traffic information system and traffic management system, providing realtime and effective information for travelers. In this paper, based on the short-term traffic-flow system, which has basic dynamic characteristics and a high degree of uncertainty, 


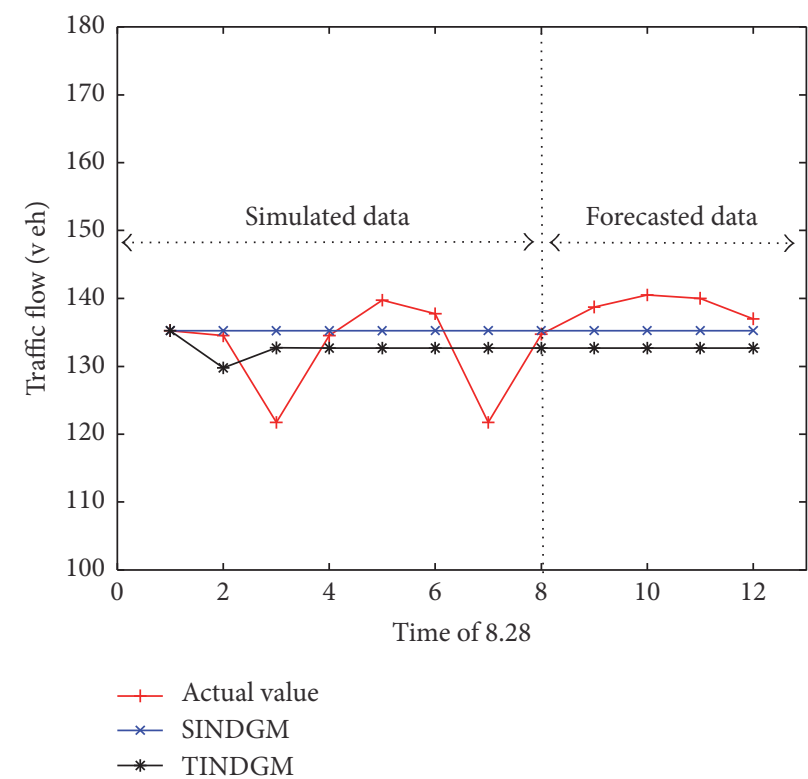

(a) The simulated and forecasted curves of the congested flow (smooth traffic) on August 28

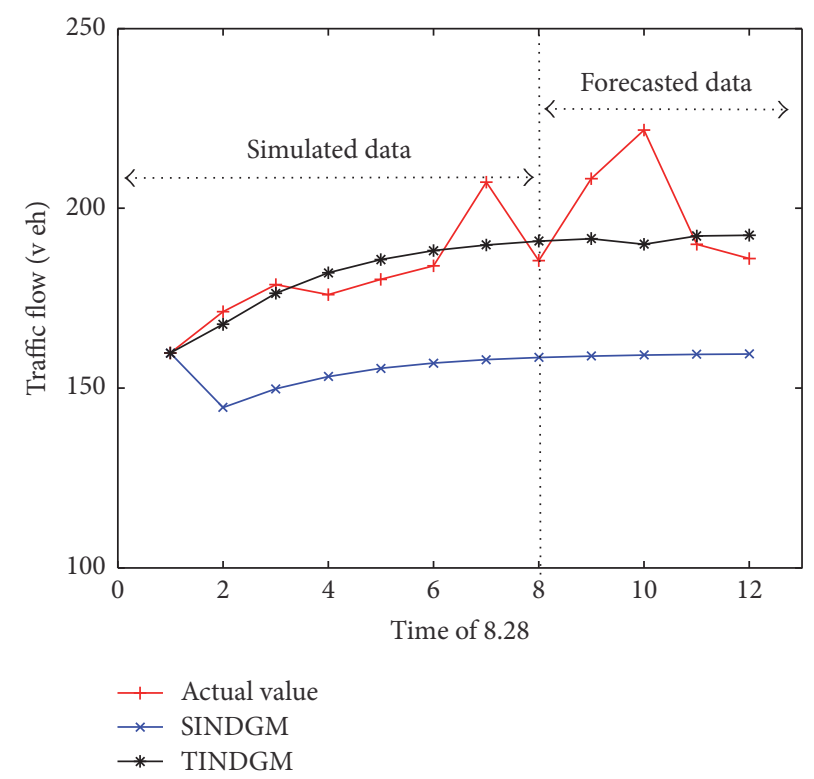

(b) The simulated and forecasted curves of the congested flow on August 28

Figure 3

the traffic-flow data, which consist of the distance, speed, and acceleration, the force synthesis and decomposition of mechanical properties, and the use of mechanical properties in the classic gray model NDGM, four types of INDGM are obtained, and the inertia model is selected according to the state of traffic flow to simulate and forecast the traffic flow. The following main conclusions can be drawn from the results:

(1) According to the dynamic characteristics of traffic flow, the basic properties of mechanics are extended to the traffic-flow data column. The approach is similar to the nonhomogenous discrete model. Using representations of the structure parameter and component parameter, four types of inertial INDGM are proposed.

(2) Through the process of obtaining the four INDGM by the force decomposition of the data, the modeling mechanism of the classical NDGM can be explained from the perspective of data decomposition. At the same time, the important properties of four INDGM are obtained.

(3) For the traffic flow at a junction in Canada, according to the traffic-flow state, the optimal INDGM is selected, and better traffic-flow prediction results are achieved.

\section{Conflicts of Interest}

The authors declare that there are no conflicts of interest regarding the publication of this paper.

\section{Acknowledgments}

This work was supported by National Natural Science Foundation of China (Grants nos. 71540027, 51479151, and 61472056), Chongqing Frontier and Applied Basic Research Project (Grant no. cstc2015jcyjA00034), and the Science and Technology Research Program of Chongqing Municipal Educational Committee (Grant no. KJ1600425).

\section{References}

[1] B. S. Kerner, "Three-phase traffic theory and highway capacity," Physica A: Statistical Mechanics and Its Applications, vol. 333, pp. 379-440, 2004.

[2] B. S. Kerner, S. L. Klenov, and A. Hiller, "Empirical test of a microscopic three-phase traffic theory," Nonlinear Dynamics, vol. 49, no. 4, pp. 525-553, 2007.

[3] V. Zyryanov, V. Kocherga, and I. Topilin, "Investigation of dependencies between parameters of two-component models of the kinetic theory of traffic flow and traffic characteristics," Transportation Research Procedia, vol. 20, pp. 746-750, 2017.

[4] M. Bouadi, K. Jetto, A. Benyoussef, and A. Kenz, "The effect of lateral interaction on traffic flow," Physica A: Statistical Mechanics and Its Applications, vol. 460, pp. 76-87, 2016.

[5] G. Chen, Z. Y. Dong, D. J. Hill, and Y. S. Xue, "Exploring reliable strategies for defending power systems against targeted attacks," IEEE Transactions on Power Systems, vol. 26, no. 3, pp. 10001009, 2011.

[6] D. Wild, "Short-term forecasting based on a transformation and classification of traffic volume time series," International Journal of Forecasting, vol. 13, no. 1, pp. 63-72, 1997.

[7] M. Cetin and G. Comert, "Short-term traffic flow prediction with regime switching models," Transportation Research Record, no. 1965, pp. 23-31, 2006. 
[8] G. A. Davis and N. L. Nihan, "Nonparametric regression and short-term freeway traffic forecasting," Journal of Transportation Engineering, vol. 117, no. 2, pp. 178-188, 1991.

[9] L. Bin-sheng, X. Zhan-wen, Y. Hai-tao, and H. Yu-peng, "A combination predicted model of short term traffic flow," in Proceedings of the International Conference on Management Science and Engineering, pp. 2075-2080, Lille, France, October 2006.

[10] B. Yao, C. Chen, Q. Cao et al., "Short-term traffic speed prediction for an urban corridor," Computer-Aided Civil and Infrastructure Engineering, vol. 32, no. 2, pp. 154-169, 2016.

[11] A. Cheng, X. Jiang, Y. Li, C. Zhang, and H. Zhu, "Multiple sources and multiple measures based traffic flow prediction using the chaos theory and support vector regression method," Physica A: Statistical Mechanics and Its Applications, vol. 466, pp. 422-434, 2017.

[12] Y. Rajabzadeh, A. H. Rezaie, and H. Amindavar, "Short-term traffic flow prediction using time-varying Vasicek model," Transportation Research Part C: Emerging Technologies, vol. 74, pp. 168-181, 2017.

[13] H. Wang, L. Liu, S. Dong, Z. Qian, and H. Wei, "A novel work zone short-term vehicle-type specific traffic speed prediction model through the hybrid EMD-ARIMA framework," Transportmetrica B, vol. 4, no. 3, pp. 159-186, 2016.

[14] Z. Zhu, B. Peng, C. Xiong, and L. Zhang, "Short-term traffic flow prediction with linear conditional Gaussian Bayesian network," Journal of Advanced Transportation, vol. 50, no. 6, pp. 1111-1123, 2016.

[15] W. Zhang, J. Tang, H. Kristian, Y. Zou, and Y. Wang, "Hybrid short-term prediction of traffic volume at ferry terminal based on data fusion," IET Intelligent Transport Systems, vol. 10, no. 8, pp. 524-534, 2016.

[16] X. Ma, Z. Tao, Y. Wang, H. Yu, and Y. Wang, "Long shortterm memory neural network for traffic speed prediction using remote microwave sensor data," Transportation Research Part C: Emerging Technologies, vol. 54, pp. 187-197, 2015.

[17] Y. Qi and S. Ishak, "A hidden Markov model for short term prediction of traffic conditions on freeways," Transportation Research Part C: Emerging Technologies, vol. 43, pp. 95-111, 2014.

[18] A. Bezuglov and G. Comert, "Short-term freeway traffic parameter prediction: Application of grey system theory models," Expert Systems with Applications, vol. 62, pp. 284-292, 2016.

[19] B. L. Smith and M. J. Demetsky, "Traffic flow forecasting: comparison of modeling approaches," Journal of Transportation Engineering, vol. 123, no. 4, pp. 261-266, 1997.

[20] E. I. Vlahogianni, M. G. Karlaftis, and J. C. Golias, "Shortterm traffic forecasting: where we are and where we're going," Transportation Research Part C: Emerging Technologies, vol. 43, part 1, pp. 3-19, 2014.

[21] J. L. Deng, Estimate and Decision of Grey System, Huazhong University of Science and Technology Press, Wuhan, China, 2002.

[22] S. F. Liu and Y. Lin, Grey Systems Theory and Applications, Springer, Berlin, Germany, 2010.

[23] H. Guo, X. Xiao, and J. Forrest, "A research on a comprehensive adaptive grey prediction model CAGM $(1, N)$," Applied Mathematics and Computation, vol. 225, pp. 216-227, 2013.

[24] X.-W. Ren, Y.-Q. Tang, J. Li, and Q. Yang, "A prediction method using grey model for cumulative plastic deformation under cyclic loads," Natural Hazards, vol. 64, no. 1, pp. 441-457, 2012.
[25] L. Wu, S. Liu, and Y. Yang, "Grey double exponential smoothing model and its application on pig price forecasting in China," Applied Soft Computing Journal, vol. 39, pp. 117-123, 2016.

[26] X. P. Xiao and S. H. Mao, Grey Forecating and Decision Methods, Science Press, Beijing, China, 2013.

[27] L. Wu, S. Liu, and Y. Yang, "A gray model with a time varying weighted generating operator," IEEE Transactions on Systems, Man, and Cybernetics: Systems, vol. 46, no. 3, pp. 427-433, 2016.

[28] B. Zeng, G. Chen, and S.-f. Liu, "A novel interval grey prediction model considering uncertain information," Journal of the Franklin Institute. Engineering and Applied Mathematics, vol. 350, no. 10, pp. 3400-3416, 2013.

[29] G. Chen, Z. Y. Dong, D. J. Hill, G. H. Zhang, and K. Q. Hua, "Attack structural vulnerability of power grids: a hybrid approach based on complex networks," Physica A: Statistical Mechanics and Its Applications, vol. 389, no. 3, pp. 595-603, 2010.

[30] L. L. Pei, W. M. Chen, and J. H. Bai, “The improved GM $(1, N)$ models with optimal background values: a case study of Chinese high-tech industry," Journal of Grey System, vol. 27, no. 3, pp. 223-233, 2015.

[31] S. H. Mao, M. Y. Gao, and X. P. Xiao, "Fractional order accumulation time-lag $\operatorname{GM}(1, N, t)$ model and its application," Systems Engineering-Theory \& Practice, vol. 35, no. 2, pp. 430436, 2015.

[32] B. Zeng, W. Meng, and M. Tong, "A self-adaptive intelligence grey predictive model with alterable structure and its application," Engineering Applications of Artificial Intelligence, vol. 50, pp. 236-244, 2016.

[33] N.-m. Xie and S.-f. Liu, "Discrete grey forecasting model and its optimization," Applied Mathematical Modelling, vol. 33, no. 2, pp. 1173-1186, 2009.

[34] N.-M. Xie, S.-F. Liu, Y.-J. Yang, and C.-Q. Yuan, “On novel grey forecasting model based on non-homogeneous index sequence," Applied Mathematical Modelling, vol. 37, no. 7, pp. 5059-5068, 2013.

[35] J. L. Deng, Grey System Makings Theory, Science Press, Beijing, China, 2014. 


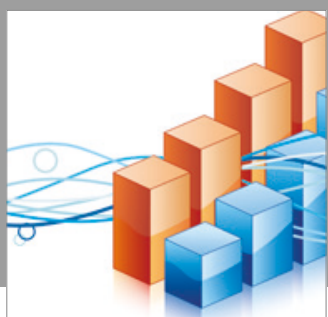

Advances in

Operations Research

vatersals

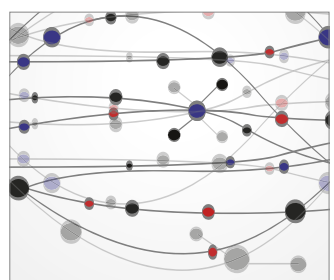

\section{The Scientific} World Journal
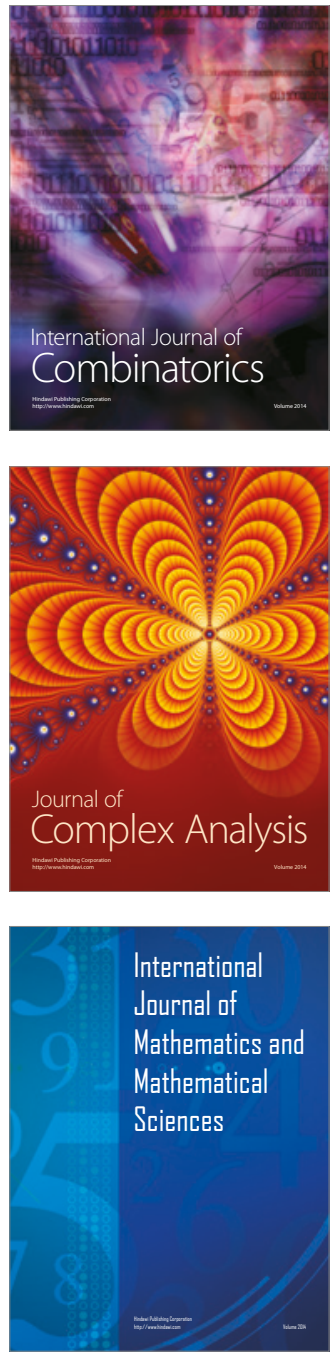
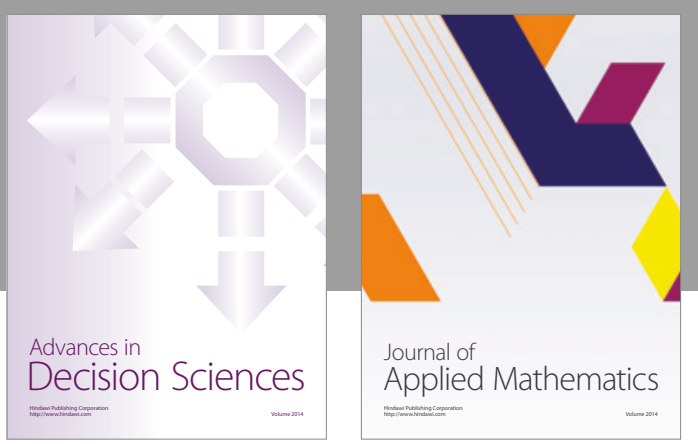

Algebra

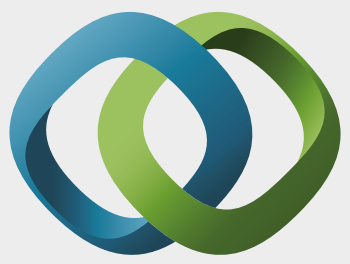

\section{Hindawi}

Submit your manuscripts at

https://www.hindawi.com
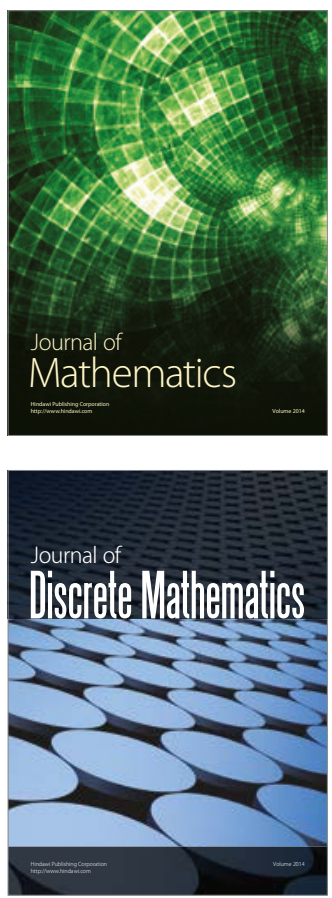

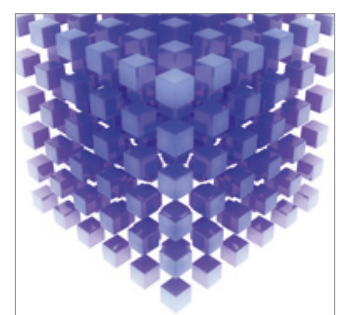

Mathematical Problems in Engineering
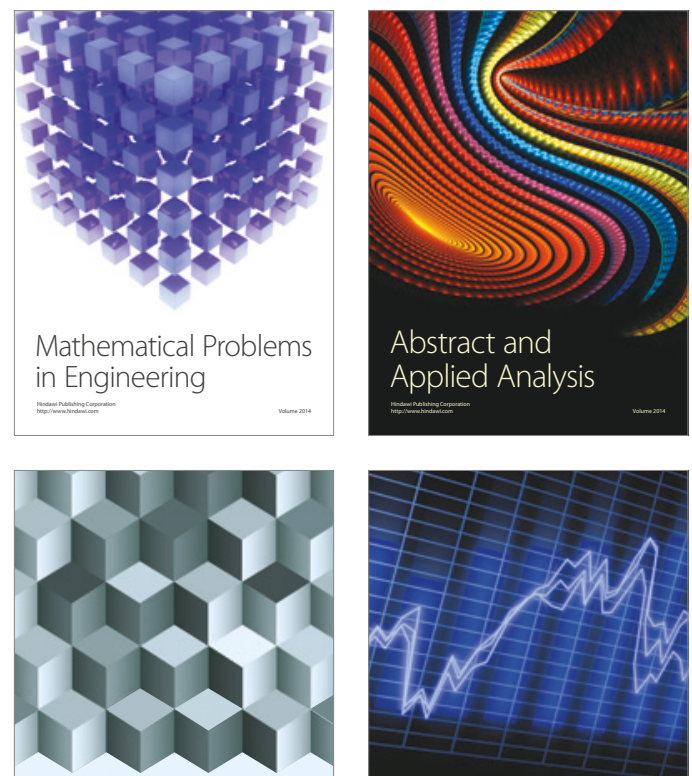

Journal of

Function Spaces

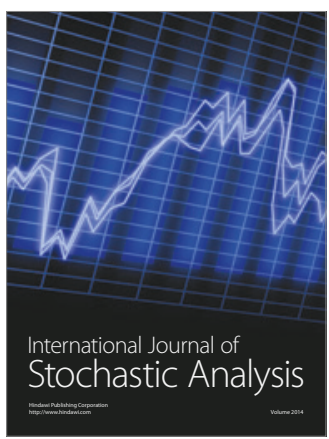

Probability and Statistics
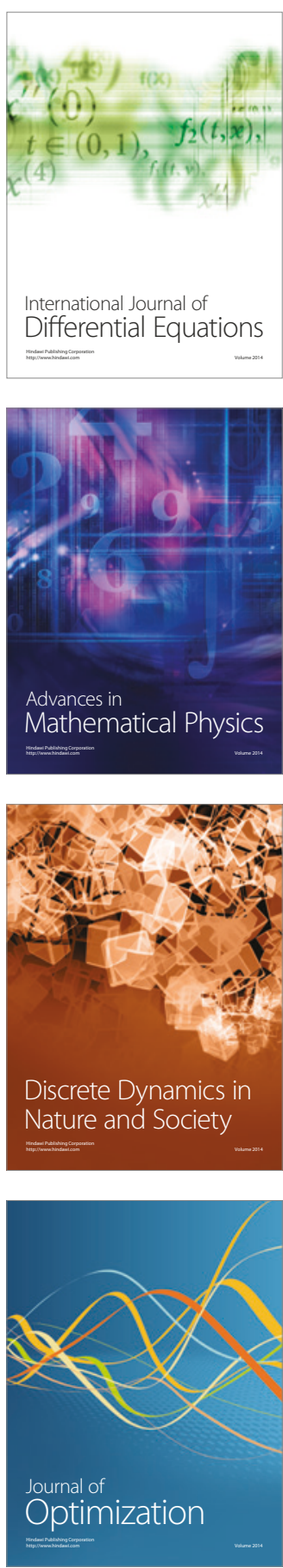